\title{
Avifaune des champs de riz de la zone humide de Grand-Bassam (Côte d'Ivoire)
}

\author{
Saint Guillaume K. ODOUKPE et Hilaire K. YAOKOKORE-BEIBRO* \\ Laboratoire de Zoologie et de Biologie Animale, Université de Cocody-Abidjan, \\ 22 B.P. 582 Abidjan 22, Côte d'Ivoire. \\ *Auteur correspondant ; E-mail: hyaokokore@yahoo.fr ; Tel : +22507634265
}

\section{RÉSUMÉ}

Les oiseaux des champs de riz de la zone humide de Grand-Bassam ont été inventoriés de janvier 2011 à février 2013 en vue de déterminer la composition et la dynamique des populations de l'avifaune de ces agroécosystèmes rizicoles. Les inventaires ornithologiques effectués dans les champs de riz par stade phénologique ont permis d'identifier 100 espèces qui utilisent directement les champs de riz pour leurs divers besoins biologiques. Ce peuplement estimé à 40556 individus d'oiseaux au cours d'un cycle de riz est beaucoup plus important à la phase de remplissage et de maturation du riz. Cette dominance du peuplement d'oiseaux à ces phases est la résultante de l'augmentation des populations d'espèces d'oiseaux granivores au détriment des autres groupes trophiques. Pourtant, en début de culture, les champs de riz regroupent toutes les catégories trophiques d'oiseaux dans des proportions sensiblement identiques.

(C) 2014 International Formulae Group. All rights reserved.

Mots clés : Biodiversité, oiseaux, rizières, site Ramsar, Grand-Bassam.

\section{INTRODUCTION}

Le riz est la principale source d'alimentation de plus de la moitié de la population mondiale (Ahmadi et Bouman, 2013) et les rizières couvrent plus de $1 \%$ de la superficie totale de la surface terrestre (Maclean et al., 2002). Malheureusement, la création et l'aménagement des rizières contribuent fréquemment à la perte des zones humides naturelles dans le monde entier (Cho, 2007). Mais une fois que les zones humides ont été drainées dans une région, les rizières fournissent le meilleur habitat qui reste pour les espèces des zones humides (Elphick, 2010). En effet, les rizières inondées sont capables de fournir un habitat adequat pour de nombreuses espèces d'oiseaux d'eau (Eadie et al., 2008 ; Nachuha, 2009 ; Elphick, 2000 ; Elphick et al., 2010 ; Wymenga et Zwarts, 2010 ; Toral et al., 2011). Elles produisent une multitude de ressources alimentaires qui sont utilisées par les oiseaux (Baouab, 2008). Ainsi, les agroécosystèmes rizicoles ont un intérêt pour les recherches écologiques sur l'avifaune (Elphick, 2010). En effet, les recherches sont menées dans le but de la résolution des conflits entre l'agriculture et les objectifs de la conservation (Ormerod et al., 2003). Mais depuis quelques années, elles s'intéressent surtout aux fonctions de conservation que jouent les rizières pour les oiseaux d'eau et à la menace que sont les 
rizières pour les zones humides naturelles (Elphick, 2010). Cependant, en Côte d'Ivoire, aucune étude ornithologique faisant même l'état des oiseaux rencontrés dans les rizières n'a été menée. Pour combler ce déficit et contribuer à la compréhension de certains phénomènes écologiques relatifs à l'association agroécosystèmes rizicoles et avifaune, une étude ornithologique s'est penchée sur la question de janvier 2011 à février 2013 dans la zone humide de GrandBassam. L'étude vise à inventorier les Oiseaux des champs de riz de cette zone humide, à en donner la composition spécifique et la dynamique des populations au cours du cycle rizicole.

\section{MATERIEL ET METHODES}

\section{Site d'étude}

Le site d'étude est inclus dans la zone humide de Grand-Bassam et est délimité dans sa longueur par les coordonnées géographiques ci-après : $5^{\circ} 13^{\prime} \mathrm{N} / 03^{\circ} 42^{\prime} \mathrm{W}$ et $5^{\circ} 17^{\prime} \mathrm{N} / 3^{\circ} 41^{\prime} \mathrm{W}$. dans sa largeur, il est naturellement délimité par la lagune Ebrié et le fleuve Comoé (Figure 1). C'est donc une zone marécageuse qui subit, pendant une longue période de l'année, les crues de ces cours d'eau. Ce phénomène s'accentue pendant la période de septembre à novembre, période au cours de laquelle les pistes villageoises sont immergées. Selon Brou (1997), le climat est de type tropical humide et caractérisé par deux saisons pluvieuses (avril à mi-juillet et mi-septembre à novembre) et deux saisons sèches (mi-juillet à miseptembre et décembre à mars). La pluviométrie moyenne annuelle est de 2100 $\mathrm{mm}$ avec une température moyenne annuelle de $26,5^{\circ} \mathrm{C}$ et une humidité relative moyenne annuelle avoisinant $85 \%$. La région appartient au secteur littoral du grand domaine phytogéographique guinéen et est caractérisée par divers types de végétations marécageuses.
Les forêts marécageuses profondément dégradées se présentent sous la forme de longues bandes étroites parallèles au rivage lagunaire (Guillaumet et Adjanohoun 1971). La végétation dominée par toutes sortes d'herbes offre un cadre idéal pour la culture intensive du riz.

La zone humide de Grand-Bassam fait depuis quelques années l'objet de culture intense du riz. Le type de riziculture pratiqué est la culture de riz avec submersion sans maîtrise de l'eau. C'est une zone nonaménagée où les semis débutent en mars, à la grande campagne rizicole et en septembre, à la petite campagne.

\section{Matériel technique}

Le matériel d'observation se compose de paires de jumelles pour l'observation directe des oiseaux. Le matériel d'enregistrement renferme des carnets de note pour la retranscription des relevés et un appareil photographique numérique reflexe (NIKON D5000, objectif : 18-200 m) pour les prises de vue. Le matériel d'identification se compose de guides d'identification des oiseaux de l'Afrique de l'Ouest (Borrow et Demey 2001, 2008) et de disques compacts des chants et cris des oiseaux d'Afrique (Chappuis, 2000). Le matériel de géolocalisation est un appareil GPS (Map source Garmin GPS 60cs) pour la détermination des coordonnées géographiques du site d'étude. Le matériel d'indication du temps est une montre qui a servi à chronométrer le temps mis aux points d'arrêt dans les différentes rizières.

\section{Collecte des données dans les champs de riz} Depuis leur mise en place jusqu'à la récolte, les rizières offrent une multitude de ressources alimentaires aux différentes espèces d'oiseaux qui s'y rencontrent. Par la physionomie et les caractéristiques des 
habitats offerts, les rizières constituent également un lieu de nidification pour certaines espèces d'oiseaux d'eau (Wymenga et Zwarts, 2010). Ainsi, pour identifier et quantifier les oiseaux qui fréquentent ces agroécosystèmes, des stades phénologiques du riz ont été définis. Le choix de ces stades s'est fait selon les phases de culture de riz et le type d'aliments disponibles durant toute la période de culture. De ce fait, six stades d'inventaire se dégagent : le stade labour, le stade semis, le stade végétatif, le stade riz immature, le stade riz mature et le stade post-récolte.

\section{Stades d'inventaires définis}

Le labour se fait par le retournement de la terre à l'aide d'un tracteur ou d'un motoculteur jusqu'à disparition totale de la végétation existante. L'utilisation d'herbicide est quelquefois associée pour la neutralisation totale des herbes. Le stade labour défini s'étend sur toute la durée du désherbage qui dure de 1 à 14 jours en général. Dans la zone humide de Grand-Bassam, les semis se font à la volée et sans apport d'eau sur la surface à cultiver. La terre est ensuite retournée à l'aide d'un tracteur ou d'un motoculteur pour recouvrir les grains de riz jetés. La durée du stade semis correspond au temps de germination des grains de riz qui s'étend de 5 à 10 jours. Le stade végétatif se singularise par la couverture de plantules de riz sur toute la surface cultivée. Cette phase s'étend de la levée jusqu'en fin de montaison sur 60 jours minimum. Cependant, les relevés avifauniques se sont effectués majoritairement de la levée à la fin du tallage du riz. Le stade riz immature ou phase de remplissage du riz est caractérisé par l'apparition d'épis sur les talles. À ce stade, le riz est à l'état liquide ou pâteux. Il s'étend de l'épiaison à la maturation et dure environ un mois. Le début d'aspect jaune paille des rizières caractérise le stade riz mature ou stade de maturation du riz. À ce stade, les grains de riz sont solides et la récolte intervient au bout de deux à trois semaines. Le stade post-récolte est caractérisé par la récolte du riz. À ce stade, les épis sont coupés et couchés sur les parcelles lorsque la récolte se fait manuellement ou avec une moissonneuse. Quel que soit le matériel utilisé pour la récolte, les grains de riz perdus sont disséminés sur l'ensemble de la surface récoltée.

\section{Inventaire des oiseaux des rizières}

Les inventaires sont effectués par parcelle de riz de $06 \mathrm{~h} 30$ à 10h30 et de $16 \mathrm{~h} 00$ à 18 h00 (Yaokokoré-Béibro et al., 2010) avec cinq inventaires par stades phénologiques définis. Tous les oiseaux qui se posent ou qui prélèvent leur nourriture sur les surfaces cultivées sont notés comme oiseaux des rizières, y compris les insectivores qui les survolent (Baouab, 2008). Lors des inventaires, 20 minutes sont requises pour une surface de 1 ha. Lorsque les parcelles sont plus grandes, cinq minutes de plus sont requises pour chaque hectare supplémentaire. Les inventaires se sont déroulés en deux temps; l'observateur demeure statique pendant les 10 premières minutes au cours desquelles il note toutes les espèces d'oiseaux ; le reste du temps est utilisé pour marcher aux alentours praticables des parcelles afin de détecter les espèces qui se posent sur les bordures. Le dénombrement de toutes les espèces d'oiseaux s'est fait individuellement ou par estimation de groupe de 100 ou 1000 , lorsque ceux-ci étaient en grand nombre (Odoukpé et al., 2014).

La liste des espèces suit l'ordre phylogénétique de Borrow et Demey (2001 et 2008) et les noms scientifiques complets sont ceux de Bisby et al. (2008). Le statut biogéographique et l'habitat préférentiel des espèces sont indiquées (Yaokokoré-Béibro, 2001 ; Bennun et al., 1996). La caractérisation 
des espèces sur la base de leur population est de Thiollay (1986) dont les normes tiennent compte de leur fréquence relative (Fr) d'observation.

La valeur maximale des cinq inventaires par stade est retenue pour les différentes analyses statistiques. La fréquence relative (Fr), l'indice de diversité de ShannonWiener (H'), l'indice d'équitabilité (J) et l'indice de similarité de Sorensen (S) ont été calculés à l'aide du logiciel Microsoft Excel 2010.

$\mathrm{Fr}=\mathrm{n}_{\mathrm{i}} / \mathrm{N}$ avec $\mathrm{n}_{\mathrm{i}}$ : somme de tous les contacts avec l'espèce $\mathrm{i}$ à chaque stade de dénombrement; $\mathrm{N}$ : $\sum \mathrm{n}_{\mathrm{i}}$ : somme des contacts avec l'ensemble des espèces observées à chaque stade.

$\mathrm{H}^{\prime}=-\sum\left(\mathrm{n}_{\mathrm{i}} / \mathrm{N}\right) \ln \left(\mathrm{n}_{\mathrm{i}} / \mathrm{N}\right)$ (Ramade, 1994);
$\mathrm{J}=\mathrm{H}^{\prime}$ / H'max Bibby et al. (1998) avec $H^{\prime} \max =\ln (S)$ où $S$ est la richesse spécifique ; $\mathrm{S}=(2 \times \mathrm{C} /(\mathrm{A}+\mathrm{B})) \times 100$ Bibby et al. (1998) avec $C$ : nombre d'espèces communes à deux stades; $\mathrm{A}$ : nombre d'espèces du stade $\mathrm{x}$; $\mathrm{B}$ : nombre d'espèces du stade $\mathrm{y}$.

Le Modèle Linéaire Généralisé, l'analyse de variance, l'analyse de classification ascendante et l'analyse factorielle des correspondances ont été effectués au seuil de 5\% à l'aide du logiciel Statistica 7.1. Pour réaliser l'analyse factorielle des correspondances (AFC), une codification du nom des espèces a été effectuée. Les trois premières lettres du nom de genre et les deux premières de l'épithète spécifique forment un code à cinq lettres pour chaque espèce.

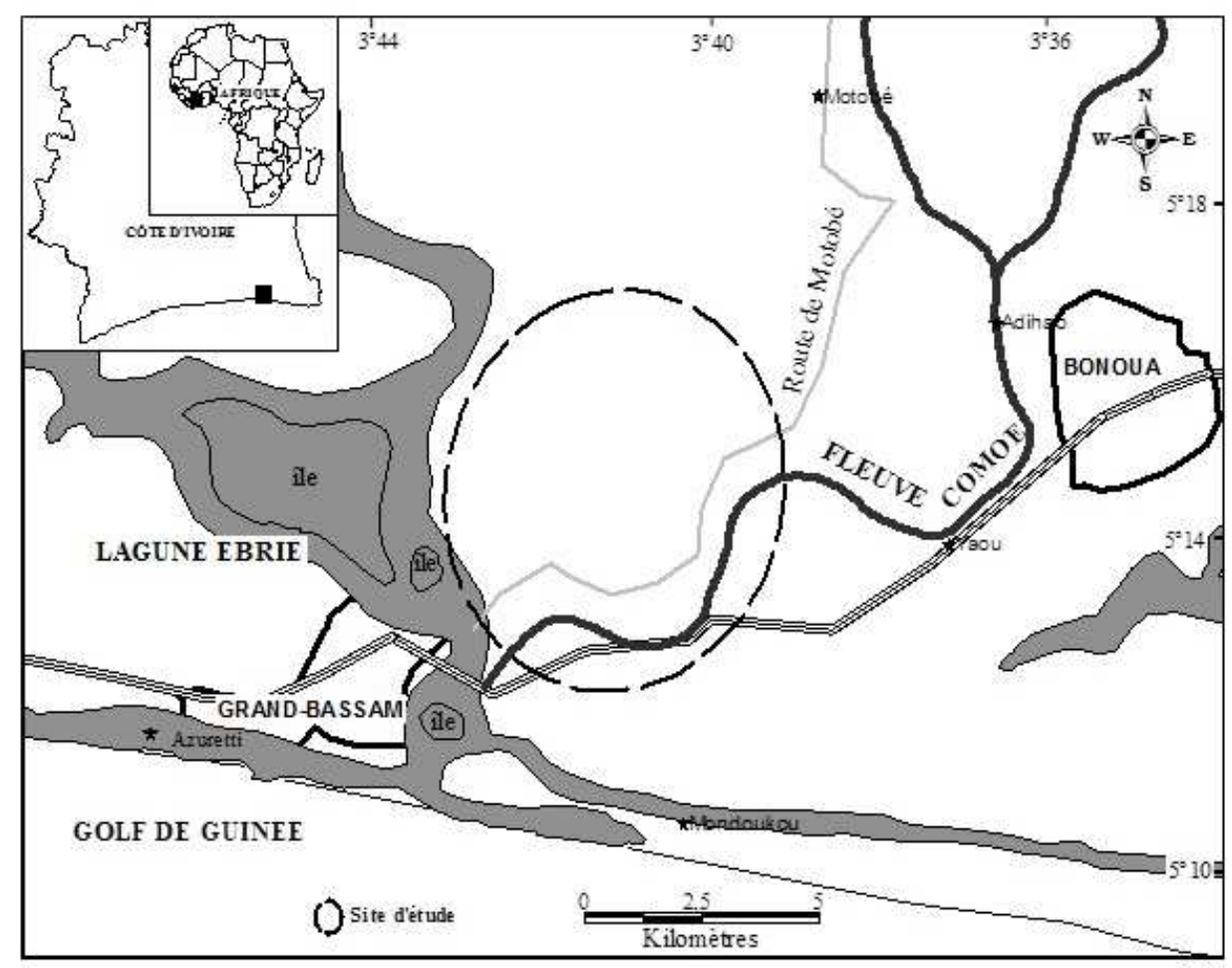

Figure 1 : Localisation du site d'étude. 


\section{RESULTATS}

Composition spécifique des oiseaux des rizières

Les observations effectuées ont permis d'identifier 100 espèces regroupées en 31 familles et 12 ordres (Tableau 1). Les familles les plus abondantes sont celles des Ardeidae et Scolopacidae avec 13 espèces chacune et les Estrildidae avec huit espèces. Les Accipitridae, Charadriidae, Alcedinidae, Hirundinidae, Sylviidae et Ploceidae suivent avec cinq espèces chacune. Le statut biogéographique indique que 49 espèces sont résidentes, 26 espèces sont migratrices et 25 espèces ont un statut mixte. Selon l'habitat préférentiel, 51 espèces sont des oiseaux des milieux ouverts, 45 espèces sont des oiseaux d'eau et quatre espèces sont des oiseaux généralistes de forêt.

\section{Abondance des populations d'oiseau des rizières}

Le peuplement d'oiseaux des rizières est estimé à 40556 individus pour une campagne. L'indice de diversité de Shannon (H') est de 2,2 avec une équitabilité de 0,48 sur l'ensemble de la superficie totale des rizières (Tableau 2). Les indices de diversité de Shannon les plus élevés s'observent à la phase végétative du riz. Les indices d'équitabilité les plus grands se perçoivent au stade labour et au stade végétatif. De toutes les espèces observées, le Tisserin gendarme Ploceus cucullatus, le Travailleur à tête rouge Quelea erythrops, l'Euplecte vorabé Euplectes afer et le Capucin nonnette Lonchura cucullata sont les espèces les plus abondantes et contribuent à $74,62 \%$ du peuplement (Tableau 3). De ce fait, elles sont les plus représentatives au cours de la phase reproductive du riz. D'autres espèces telles que le Héron garde-bœuf Bubulcus ibis, le Jacana à poitrine dorée Actophilornis africanus, la Tourterelle à collier Streptopelia semitorquata, l'Astrild à joues orange Estrilda melpoda et le Capucin pie Lonchura fringilloides sont régulières et contribuent à $13,86 \%$ du peuplement.

Au stade de préparation du sol, une charge avifaunique de 1749 individus appartenant à 51 espèces d'oiseaux a été obtenue pour une campagne rizicole. C'est le stade qui enregistre le plus faible nombre d'individus et le second indice de diversité de Shannon (H') le plus élevé qui est de 2,99 et une équitabilité de 0,76 . Les espèces les plus abondantes représentent $60,09 \%$ du peuplement et sont Bubulcus ibis, Milvus migrans, Actophilornis africanus, Streptopelia semitorquata, Euplectes afer et Lonchura cucullata. Les espèces régulières contribuent à $28,87 \%$ du peuplement et les autres espèces représentent $11,04 \%$ du peuplement.

$\mathrm{Au}$ stade des semis, une charge avifaunique de 6904 individus appartenant à 65 espèces d'oiseaux a été notée pour une campagne rizicole. L'indice de diversité de Shannon (H') est de 2,16 avec une équitabilité de 0,52. Les espèces les plus abondantes contribuent à $65,56 \%$ du peuplement et sont Actophilornis africanus, Streptopelia semitorquata et Quelea erythrops. Les espèces régulières représentent $26,36 \%$ et les autres espèces contribuent à $8,08 \%$ du peuplement.

$\mathrm{Au}$ stade végétatif, une charge avifaunique de 2833 individus appartenant à 79 espèces d'oiseaux a été obtenue pour une campagne rizicole. Ce stade enregistre le plus d'espèces et est le plus diversifié avec un indice de diversité de Shannon (H') de 3,17 et une équitabilité de 0,72 . Les espèces les plus abondantes représentent $57,85 \%$ du peuplement et sont Bubulcus ibis, Dendrocygna viduata, Actophilornis africanus, Quelea erythrops, Euplectes afer et Lonchura cucullata. Les espèces régulières et les autres espèces contribuent respectivement à $27,21 \%$ et $14,94 \%$ du peuplement. 
Au stade de riz immature, une charge avifaunique de 11718 individus appartenant à 60 espèces d'oiseaux a été obtenue pour une campagne rizicole. C'est le second stade qui enregistre le plus d'individus et marque l'indice de diversité de Shannon (H') le plus faible qui est de 1,56 avec une équitabilité de 0,38 . Les espèces les plus abondantes représentent $89,77 \%$ du peuplement et sont Ploceus cucullatus, Quelea erythrops, Euplectes afer et Lonchura cucullata. Deux autres espèces, Estrilda melpoda et Lonchura fringilloides, sont moins abondantes mais régulières à ce stade du riz.

$\mathrm{Au}$ stade de riz mature, une charge avifaunique de 11995 individus appartenant à 61 espèces d'oiseaux a été obtenue pour une campagne rizicole. Ce stade renferme le plus d'individus et enregistre le second indice de diversité de Shannon (H') le plus faible qui est de 1,64 avec une équitabilité de 0,4. Les espèces les plus abondantes représentent $89,18 \%$ du peuplement et sont Ploceus cucullatus, Quelea erythrops, Euplectes afer et Lonchura cucullata. Les espèces Estrilda melpoda et Lonchura fringilloides sont également régulières à ce stade.

$\mathrm{Au}$ stade des récoltes, une charge avifaunique de 5357 individus appartenant à 50 espèces d'oiseaux a été notée pour une campagne rizicole. L'indice de diversité de Shannon (H') est de 1,72 avec une équitabilité de 0,44 . Les espèces les plus abondantes contribuent à $84,95 \%$ du peuplement et sont Streptopelia semitorquata, Ploceus cucullatus, Euplectes afer et Lonchura cucullata. Les espèces régulières et les autres espèces contribuent respectivement à $9,17 \%$ et $5,88 \%$ du peuplement.

\section{Relation entre les stades phénologiques}

L'analyse de classification ascendante réalisée à partir de la matrice "effectifs de chaque espèce/stade phénologique du riz" présente un dendrogramme à deux ensembles A et B (Figure 2). L'ensemble A regroupe initialement le stade labour (SL) et le stade végétatif (SV) qui comptent respectivement 1749 individus de 51 espèces et 2833 individus de 79 espèces. À ce couple de stades se lient le stade post-récolte (SPR) puis le stade semis (SS) qui comptent respectivement 5357 individus de 50 espèces et 6904 individus de 65 espèces. L'ensemble B regroupe le stade riz immature (SRI) et le stade riz mature (SRM) qui comptent respectivement 11778 individus de 60 espèces et 11995 individus de 61 espèces. Les indices de similarités calculés sont élevés pour toutes les associations des stades phénologiques du riz. L'indice le plus élevé est incontestablement celui de l'association des stades SRI et SRM qui s'évalue à $87,60 \%$. De toutes les espèces d'oiseaux observées dans les champs de riz, seulement 31 espèces se rencontrent sur l'ensemble du cycle du riz.

\section{Variation de l'avifaune en fonction de la phénologie du riz}

Les courbes réalisées à partir de la matrice "effectifs du peuplement/stades phénologiques" par campagne montrent une variation nette du peuplement (Figure 3). L'analyse de variance confirme cette variation en montrant que les effectifs des espèces sont statistiquement différents $(\mathrm{ddl}=6 ; \mathrm{F}=3,6 ; \mathrm{p}$ $<0,005)$. Les courbes des deux types de campagne rizicole sont presque identiques mais les effectifs des individus pendant la grande campagne sont plus importants que ceux de la petite campagne. Les courbes présentent des flux importants au stade semis et durant toute la phase reproductive au cours de laquelle elles atteignent le maximum au stade riz mature. D'autres courbes réalisées à partir de la matrice "effectifs du peuplement/stades phénologiques" par année montrent également que le peuplement des 
oiseaux a varié au cours des stades phénologiques du riz (Figure 4). Ces courbes présentent des allures semblables entre elles et aux précédentes.

Distribution des espèces en fonction des stades phénologiques

L'analyse factorielle des correspondances effectuée à partir de la matrice "effectifs des espèces/stades phénologiques" montre qu'il y'a une variation dans la distribution des espèces au cours des stades du riz (Figure 5). En effet, le Modèle Linéaire Généralisé confirme cette observation en montrant que la distribution des espèces a varié au cours des stades ( ddl = $5 ; \mathrm{w}=219,37 ; \mathrm{p}<0,001)$. En considérant la variable stades phénologiques et en suivant l'axe 1 qui exprime 47,42\% de la variabilité, la figure présente deux ensembles. Le premier ensemble, qui se trouve du côté positif de l'axe 1, regroupe les stades SRI, SRM et SPR. En suivant l'axe 2, il est constaté que le stade SPR s'isole du côté positif de l'axe tandis que les autres stades de l'ensemble se trouvent du côté opposé. Le second ensemble, qui se trouve du côté négatif de l'axe 1, regroupe les stades SL, SS et SV. En considérant la variable espèces, Cypsiurus parvus, Hirundo rustica, Ploceus nigerrimus, Quelea erythrops, Pyrenestes ostrinus, Estrilda melpoda, Spermestes cucullatus, Spermestes bicolor etc. sont proches des stades SRI et SRM. Quant au stade SPR, ce sont Streptopelia semitorquata et Spermophaga haematina qui s'y rapprochent. Les stades SL, SS et SV sont proches des espèces Bubulcus ibis, Egretta alba, Milvus migrans, Falco ardosiaceus, Gallinula angulata, Actophilornis africana, Ceryle rudis, Anthus leucophrys, Corvus albus, Lanius collaris etc.



Figure 2 : Dendrogramme montrant les similarités entre les stades phénologiques sur la base du nombre d'individus par espèces. SL : Stade Labour ; SS : Stade Semis ; SV : Stade végétatif ; SRI : Stade Riz Immature ; SRM : Stade Riz Mature ; SPR : Stade Post-Récolte. 


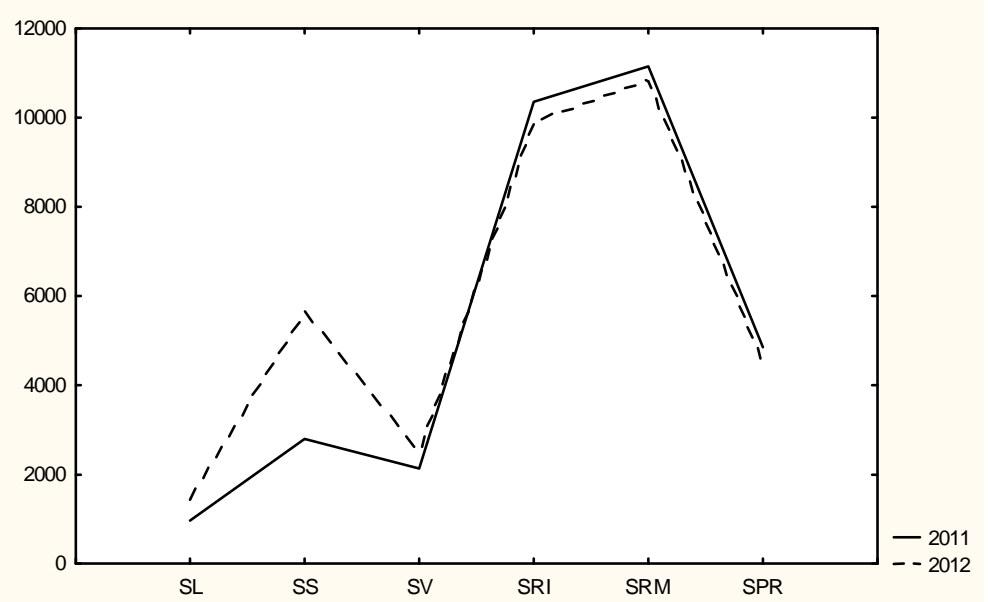

Figure 3: Variation du peuplement d'oiseaux des rizières par stades phénologiques et par campagnes. SL : Stade Labour; SS : Stade Semis ; SV : Stade végétatif ; SRI : Stade Riz Immature ; SRM : Stade Riz Mature ; SPR : Stade Post-Récolte.

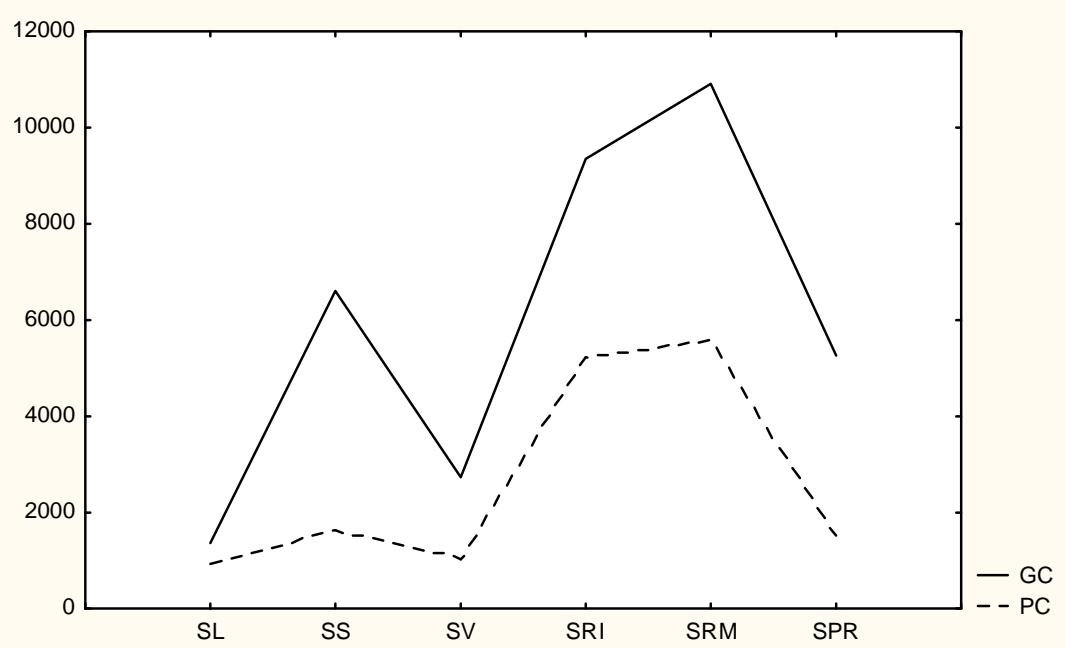

Figure 4 : Variation du peuplement d'oiseaux des rizières par stades phénologiques et par année. SL : Stade Labour ; SS : Stade Semis ; SV : Stade végétatif ; SRI : Stade Riz Immature ; SRM : Stade Riz Mature ; SPR : Stade Post-Récolte 




Figure 5 : Distribution des espèces d'oiseaux des rizières par stades phénologiques.

Tableau 1: Liste des espèces d'oiseaux observées sur les rizières de février 2011 à février 2013.

\begin{tabular}{|c|c|c|c|c|}
\hline ORDRES /Familles/Espèces & Noms français & English names & Biog & Hab \\
\hline \multicolumn{5}{|l|}{ PODICIPEDIFORMES } \\
\hline \multicolumn{5}{|l|}{ Podicipedidae } \\
\hline Tachybaptus ruficollis (Pallas, 1764) & Grèbe castagneux & Little Grebe & $\mathrm{R}$ & $\mathrm{E}$ \\
\hline \multicolumn{5}{|l|}{ PELECANIFORMES } \\
\hline \multicolumn{5}{|l|}{ Phalacrocoracidae } \\
\hline $\begin{array}{l}\text { Phalacrocorax africanus (J. F. Gmelin, } \\
\text { 1789) }\end{array}$ & Cormoran africain & Reed Cormorant & $\mathrm{R}$ & $\mathrm{E}$ \\
\hline \multicolumn{5}{|l|}{ CICONIIFORMES } \\
\hline \multicolumn{5}{|l|}{ Ardeidae } \\
\hline Ixobrychus minutus (Linné, 1766) & Blongios nain & Little Bittern & $\mathrm{R} / \mathrm{P}$ & $\mathrm{E}$ \\
\hline Nycticorax nycticorax (Linné, 1758) & Bihoreau gris & $\begin{array}{l}\text { Black-crowned } \\
\text { Night Heron }\end{array}$ & $\mathrm{R} / \mathrm{P}$ & E \\
\hline Ardeola ralloides (Scopoli, 1769) & Crabier chevelu & $\begin{array}{c}\text { Common } \\
\text { Squacco Heron }\end{array}$ & $\mathrm{R} / \mathrm{P}$ & E \\
\hline Bubulcus ibis (Linné, 1758) & Héron garde-bœufs & Cattle Egret & $\mathrm{R} / \mathrm{M}$ & $\mathrm{E}$ \\
\hline Butorides striata (Linné, 1758) & Héron strié & $\begin{array}{l}\text { Green-backed } \\
\text { Heron }\end{array}$ & $\mathrm{R}$ & $\mathrm{E}$ \\
\hline Egretta ardesiaca (Wagler, 1827) & $\begin{array}{l}\text { Aigrette ardoisée } \\
1466\end{array}$ & Black Egret & $\mathrm{R} / \mathrm{M}$ & $\mathrm{E}$ \\
\hline
\end{tabular}


S. G. K. ODOUKPE et H. K. YAOKOKORE-BEIBRO / Int. J. Biol. Chem. Sci. 8(4): 1458-1480, 2014

\begin{tabular}{|c|c|c|c|c|}
\hline ORDRES /Familles/Espèces & Noms français & English names & Biog & Hab \\
\hline Egretta gularis (Bosc, 1792) & $\begin{array}{l}\text { Aigrette à gorge } \\
\text { blanche }\end{array}$ & Reef Heron & $\mathrm{R}$ & $\mathrm{E}$ \\
\hline Egretta garzetta (Linné, 1766) & Aigrette garzette & Little Egret & $\mathrm{R} / \mathrm{M}$ & $\mathrm{E}$ \\
\hline Egretta intermedia (Wagler, 1829) & $\begin{array}{l}\text { Aigrette } \\
\text { intermédiaire }\end{array}$ & $\begin{array}{l}\text { Yellow-billed } \\
\text { Egret }\end{array}$ & $\mathrm{R} / \mathrm{M}$ & $\mathrm{E}$ \\
\hline Egretta alba (Linné, 1758) & Grande aigrette & $\begin{array}{l}\text { Great White } \\
\text { Egret }\end{array}$ & $\mathrm{R} / \mathrm{M}$ & $\mathrm{E}$ \\
\hline Ardea purpurea Linné, 1766 & Héron pourpré & Purple Heron & $\mathrm{R} / \mathrm{P}$ & $\mathrm{E}$ \\
\hline Ardea cinerea Linné, 1758 & Héron cendré & Grey Heron & $\mathrm{R} / \mathrm{P}$ & $\mathrm{E}$ \\
\hline $\begin{array}{l}\text { Ardea melanocephala Vigors \& Children, } \\
1826\end{array}$ & $\begin{array}{c}\text { Héron } \\
\text { mélanocéphale }\end{array}$ & $\begin{array}{l}\text { Black-headed } \\
\text { Heron }\end{array}$ & $\mathrm{R} / \mathrm{M}$ & $\mathrm{E}$ \\
\hline \multicolumn{5}{|l|}{ Ciconiidae } \\
\hline Ciconia episcopus (Boddaert, 1783) & Cigogne épiscopale & $\begin{array}{l}\text { Woolly-necked } \\
\text { Stork }\end{array}$ & $\mathrm{R} / \mathrm{M}$ & $\mathrm{E}$ \\
\hline \multicolumn{5}{|l|}{ Threskiornithidae } \\
\hline Plegadis falcinellus (Linné, 1766) & Ibis falcinelle & Glossy Ibis & $\mathrm{R} / \mathrm{P}$ & E \\
\hline \multicolumn{5}{|l|}{$\begin{array}{l}\text { ANSERIFORMES } \\
\text { Anatidae }\end{array}$} \\
\hline Dendrocygna viduata (Linné, 1766) & Dendrocygne veuf & $\begin{array}{l}\text { White-faced } \\
\text { Duck }\end{array}$ & $\mathrm{R} / \mathrm{M}$ & $\mathrm{E}$ \\
\hline Plectropterus gambensis (Linné, 1766) & $\begin{array}{l}\text { Oie-armée de } \\
\text { Gambie }\end{array}$ & $\begin{array}{l}\text { Spur-winged } \\
\text { Goose }\end{array}$ & $\mathrm{R}$ & $\mathrm{E}$ \\
\hline Nettapus auritus (Boddaert, 1783) & Sarcelle à oreillons & Pygmy Goose & $\mathrm{R}$ & $\mathrm{E}$ \\
\hline \multicolumn{5}{|l|}{$\begin{array}{l}\text { FALCONIFORMES } \\
\text { Accipitridae }\end{array}$} \\
\hline Elanus caeruleus (Desfontaines, 1789) & Elanion blanc & $\begin{array}{l}\text { Black-shouldered } \\
\text { Kite }\end{array}$ & $\mathrm{R}$ & f. \\
\hline Milvus migrans (Boddaert, 1783) & Milan noir & Black Kite & $\mathrm{M} / \mathrm{P}$ & f. \\
\hline Circus pygargus (Linné, 1758) & Busard cendré & $\begin{array}{l}\text { Montagu's } \\
\text { Harrier }\end{array}$ & $\mathrm{P}$ & f. \\
\hline Circus aeruginosus (Linné, 1758) & Busard des roseaux & $\begin{array}{l}\text { Eurasian Marsh- } \\
\text { Harrier }\end{array}$ & $\mathrm{P}$ & f. \\
\hline Accipiter badius (J. F. Gmelin, 1788) & Epervier shikra & Shikra & $\mathrm{R} / \mathrm{M}$ & f. \\
\hline \multicolumn{5}{|l|}{ Falconidae } \\
\hline Falco ardosiaceus Vieillot, 1823 & Faucon ardoisé & Grey Kestrel & $\mathrm{R}$ & f. \\
\hline \multicolumn{5}{|l|}{ GRUIFORMES } \\
\hline Crex egregia (Peters, 1854) & Râle des prés & African Crake & $\mathrm{R} / \mathrm{M}$ & f. \\
\hline Amaurornis flavirostra (Swainson, 1837) & Râle à bec jaune & Blake Crake & $\mathrm{R}$ & $\mathrm{E}$ \\
\hline Porphyrio alleni Thomson, 1842 & Talève d'Allen & Lesser Gallinule & $\mathrm{R} / \mathrm{M}$ & $\mathrm{E}$ \\
\hline Gallinula angulata Sundevall, 1850 & Gallinule africaine & Lesser Moorhen & $\mathrm{R} / \mathrm{M}$ & $\mathrm{E}$ \\
\hline \multicolumn{5}{|l|}{ Gruidae } \\
\hline Balearica pavonina (Linné, 1758) & Grue couronnée & $\begin{array}{c}\text { Northern } \\
\text { Crowned Crane }\end{array}$ & Oc & f. \\
\hline
\end{tabular}




\begin{tabular}{|c|c|c|c|c|}
\hline ORDRES /Familles/Espèces & Noms français & English names & Biog & Hab \\
\hline \multicolumn{5}{|l|}{ CHARADRIIFORMES } \\
\hline \multicolumn{5}{|l|}{ Jacanidae } \\
\hline $\begin{array}{l}\text { Actophilornis africanus (J. F. Gmelin, } \\
\text { 1789) }\end{array}$ & $\begin{array}{c}\text { Jacana à poitrine } \\
\text { dorée }\end{array}$ & African Jacana & $\mathrm{R}$ & E \\
\hline \multicolumn{5}{|l|}{ Rostratulidae } \\
\hline Rostratula benghalensis (Linné, 1758) & Rhynchée peinte & Painted Snipe & $\mathrm{R} / \mathrm{M}$ & $\mathrm{E}$ \\
\hline \multicolumn{5}{|l|}{ Charadriidae } \\
\hline Charadrius hiaticula Linné, 1758 & $\begin{array}{l}\text { Pluvier grand- } \\
\text { gravelot }\end{array}$ & Ringed Plover & $\mathrm{P}$ & $\mathrm{E}$ \\
\hline Charadrius forbesi (Shelley, 1883) & Pluvier de Forbes & Forbes's Plover & $\mathrm{R} / \mathrm{M}$ & $\mathrm{E}$ \\
\hline Pluvialis dominica (Muller, 1776) & Pluvier bronzé & $\begin{array}{l}\text { American Golden } \\
\text { Plover }\end{array}$ & Oc & $\mathrm{E}$ \\
\hline Vanellus senegallus (Linné, 1766) & Vanneau du Sénégal & Wattled Plover & $\mathrm{R} / \mathrm{M}$ & E \\
\hline Vanellus spinosus (Linné, 1758) & Vanneau à éperons & $\begin{array}{l}\text { Spur-winged } \\
\text { Plover }\end{array}$ & $\mathrm{P}$ & E \\
\hline \multicolumn{5}{|l|}{ Scolopacidae } \\
\hline Calidris canutus (Linné, 1758) & Bécasseau maubèche & Red Knot & $\mathrm{P}$ & $\mathrm{E}$ \\
\hline Calidris minuta (Leisler, 1812) & Bécasseau minute & Little Stint & $\mathrm{P}$ & $\mathrm{E}$ \\
\hline Calidris fuscicollis (Vieillot, 1819) & $\begin{array}{l}\text { Bécasseau à } \\
\text { croupion blanc }\end{array}$ & $\begin{array}{l}\text { White-Rumped } \\
\text { Sandpiper }\end{array}$ & $\mathrm{Oc}$ & $\mathrm{E}$ \\
\hline Calidris melanotos (Vieillot, 1819) & $\begin{array}{l}\text { Bécasseau à poitrine } \\
\text { cendrée }\end{array}$ & $\begin{array}{l}\text { Pectoral } \\
\text { Sandpiper }\end{array}$ & $\mathrm{Oc}$ & $\mathrm{E}$ \\
\hline Calidris ferruginea (Pontoppidan, 1763) & Bécasseau cocorli & Curlew Sandpiper & $\mathrm{P}$ & $\mathrm{E}$ \\
\hline Gallinago gallinago (Linné, 1758) & Bécassine des marais & Common Snipe & $\mathrm{P}$ & $\mathrm{E}$ \\
\hline Numenius phaeopus (Linné, 1758) & Courlis corlieu & Whimbrel & $\mathrm{P}$ & $\mathrm{E}$ \\
\hline Tringa totanus (Linné, 1758) & Chevalier gambette & Redshank & $\mathrm{P}$ & $\mathrm{E}$ \\
\hline Tringa stagnatilis (Bechstein, 1803) & Chevalier stagnatile & Marsh Sandpiper & $\mathrm{P}$ & $\mathrm{E}$ \\
\hline Tringa nebularia (Gunnerus, 1767) & Chevalier aboyeur & Greenshank & $\mathrm{P}$ & $\mathrm{E}$ \\
\hline Tringa ochropus Linné, 1758 & Chevalier cul-blanc & Green Sandpiper & $\mathrm{P}$ & $\mathrm{E}$ \\
\hline Tringa glareola (Linné, 1758) & Chevalier sylvain & Wood Sandpiper & $\mathrm{P}$ & $\mathrm{E}$ \\
\hline Actitis hypoleucos (Linné, 1758) & Chevalier guignette & $\begin{array}{l}\text { Common } \\
\text { Sandpiper }\end{array}$ & $P$ & $\mathrm{E}$ \\
\hline
\end{tabular}

\section{COLUMBIFORMES}

\section{Columbidae}

Turtur tympanistria (Temminck, 1809)

Turtur afer (Linné, 1766)

Streptopelia semitorquata (Rüppell, 1837)

Streptopelia senegalensis (Linné, 1766)

CUCULIFORMES

Cuculidae

Chrysococcyx caprius (Boddaert, 1783)

Centropus grillii Hartlaub, 1861

Coucou didric

Coucal de Grill

$\begin{array}{cccc}\begin{array}{c}\text { Tourtelette } \\ \text { tambourette } \\ \begin{array}{c}\text { Tourtelette } \\ \text { améthystine }\end{array}\end{array} & \begin{array}{c}\text { Tambourine } \\ \text { Dove } \\ \text { Blue-spotted } \\ \text { Wood Dove }\end{array} & \text { R } & \text { F } \\ \text { fourterelle à collier } & \text { Red-eyed Dove } & \text { R } & \text { f. } \\ \text { ourterelle maillée } & \text { Laughing Dove } & \text { R } & \text { f. }\end{array}$


S. G. K. ODOUKPE et H. K. YAOKOKORE-BEIBRO / Int. J. Biol. Chem. Sci. 8(4): 1458-1480, 2014

\begin{tabular}{|c|c|c|c|c|}
\hline ORDRES /Familles/Espèces & Noms français & English names & Biog & Hab \\
\hline Centropus senegalensis (Linné, 1766) & Coucal du Sénégal & Senegal Coucal & $\mathrm{R}$ & f. \\
\hline \multirow{2}{*}{\multicolumn{5}{|c|}{$\begin{array}{l}\text { APODIFORMES } \\
\text { Apodidae }\end{array}$}} \\
\hline & & & & \\
\hline Cypsiurus parvus (Lichtenstein, 1823) & $\begin{array}{l}\text { Martinet des } \\
\text { palmiers }\end{array}$ & $\begin{array}{l}\text { African Palm- } \\
\text { Swift }\end{array}$ & $\mathrm{R}$ & f. \\
\hline Apus affinis (J. E. Gray, 1830) & Martinet des maisons & Little Swift & $\mathrm{R}$ & f. \\
\hline \multirow{2}{*}{\multicolumn{5}{|c|}{$\begin{array}{l}\text { CORACIIFORMES } \\
\text { Alcedinidae }\end{array}$}} \\
\hline & & & & \\
\hline Halcyon malimbica (Shaw, 1811) & $\begin{array}{l}\text { Martin-chasseur à } \\
\text { poitrine bleue }\end{array}$ & $\begin{array}{l}\text { Blue-breasted } \\
\text { Kingfisher }\end{array}$ & $\mathrm{R}$ & $\mathrm{F}$ \\
\hline Halcyon senegalensis (Linné, 1766) & $\begin{array}{l}\text { Martin-chasseur du } \\
\text { Sénégal }\end{array}$ & $\begin{array}{l}\text { Woodland } \\
\text { Kingfisher }\end{array}$ & $\mathrm{R}$ & f. \\
\hline Ceyx pictus (Boddaert, 1783) & $\begin{array}{l}\text { Martin-pêcheur } \\
\text { pygmée }\end{array}$ & $\begin{array}{l}\text { Pygmy } \\
\text { Kingfisher }\end{array}$ & $\mathrm{R} / \mathrm{M}$ & f. \\
\hline Alcedo cristata Pallas, 1764 & $\begin{array}{l}\text { Martin-pêcheur } \\
\text { huppé }\end{array}$ & $\begin{array}{l}\text { Malachite } \\
\text { Kingfisher }\end{array}$ & $\mathrm{R}$ & E \\
\hline Ceryle rudis (Linné, 1758) & Martin-pêcheur pie & Pied Kingfisher & $\mathrm{R}$ & E \\
\hline \multicolumn{5}{|l|}{ Meropidae } \\
\hline Merops pusillus Muller, 1776 & Guêpier nain & Little Bee-eater & $\mathrm{R}$ & f. \\
\hline \multicolumn{5}{|l|}{ PASSERIFORMES } \\
\hline Hirundinidae & & & & \\
\hline Riparia riparia (Linné, 1758) & Hirondelle de rivage & $\begin{array}{l}\text { European Sand } \\
\text { Martin }\end{array}$ & $\mathrm{P}$ & f. \\
\hline Hirundo semirufa Sundevall, 1850 & $\begin{array}{l}\text { Hirondelle à ventre } \\
\text { roux }\end{array}$ & $\begin{array}{l}\text { Red-breasted } \\
\text { Swallow }\end{array}$ & $\mathrm{R}$ & f. \\
\hline $\begin{array}{l}\text { Hirundo abyssinica Guerin-Meneville, } \\
1843\end{array}$ & Hirondelle striée & $\begin{array}{l}\text { Lesser Striped } \\
\text { Swallow }\end{array}$ & $\mathrm{R} / \mathrm{M}$ & f. \\
\hline Hirundo aethiopica Blanford, 1869 & Hirondelle d'Ethiopie & $\begin{array}{l}\text { Ethiopian } \\
\text { Swallow }\end{array}$ & $\mathrm{R} / \mathrm{M}$ & f. \\
\hline Hirundo rustica Linné, 1758 & Hirondelle rustique & Barn Swallow & $\mathrm{P}$ & f. \\
\hline \multicolumn{5}{|l|}{ Motacillidae } \\
\hline Motacilla flava Linné, 1758 & $\begin{array}{l}\text { Bergeronnette } \\
\text { printanière }\end{array}$ & Yellow Wagtail & $\mathrm{P}$ & f. \\
\hline Anthus leucophrys Vieillot, 1818 & Pipit à dos uni & $\begin{array}{l}\text { Plain-backed } \\
\text { Pipit }\end{array}$ & $\mathrm{R}$ & f. \\
\hline Macronyx croceus (Vieillot, 1816) & $\begin{array}{l}\text { Sentinelle à gorge } \\
\text { jaune }\end{array}$ & $\begin{array}{l}\text { Yellow-throated } \\
\text { Longclaw }\end{array}$ & $\mathrm{R}$ & f. \\
\hline
\end{tabular}

Pycnonotus barbatus (Desfontaines, 1789)

Bulbul des jardins Garden Bulbul R f.

Turdidae

Saxicola rubetra (Linné, 1758)

Tarier des prés

Whinchat

P f.

Sylviidae

Acrocephalus schoenobaenus (Linné, 1758)

Phragmite des joncs Sedge Warbler P f. 
S. G. K. ODOUKPE et H. K. YAOKOKORE-BEIBRO / Int. J. Biol. Chem. Sci. 8(4): 1458-1480, 2014

\begin{tabular}{|c|c|c|c|c|}
\hline ORDRES /Familles/Espèces & Noms français & English names & Biog & Hab \\
\hline Cisticola erythrops (Hartlaub, 1857) & $\begin{array}{l}\text { Cisticole à face } \\
\text { rousse }\end{array}$ & $\begin{array}{l}\text { Red-faced } \\
\text { Cisticola }\end{array}$ & $\mathrm{R}$ & f. \\
\hline Cisticola galactotes (Temminck, 1821) & Cisticole roussâtre & $\begin{array}{l}\text { Winding } \\
\text { Cisticola }\end{array}$ & $\mathrm{R}$ & f. \\
\hline Cisticola brachypterus (Sharpe, 1870) & $\begin{array}{l}\text { Cisticole à ailes } \\
\text { courtes }\end{array}$ & Siffling Cisticola & $\mathrm{R}$ & f. \\
\hline Prinia subflava (J. F. Gmelin, 1789) & Prinia modeste & $\begin{array}{c}\text { Tawny-flanked } \\
\text { Prinia }\end{array}$ & $\mathrm{R}$ & f. \\
\hline \multicolumn{5}{|l|}{ Nectariniidae } \\
\hline Cinnyris cupreus (Shaw, 1812) & Souimanga cuivré & Copper Sunbird & $\mathrm{R}$ & f. \\
\hline \multicolumn{5}{|l|}{ Laniidae } \\
\hline Lanius collaris Linné, 1766 & Pie-grièche fiscale & Fiscal Shrike & $\mathrm{R}$ & f. \\
\hline \multicolumn{5}{|l|}{ Corvidae } \\
\hline Corvus albus Muller, 1776 & Corbeau pie & Pied Crow & $\mathrm{R}$ & f. \\
\hline \multicolumn{5}{|l|}{ Passeridae } \\
\hline Passer domesticus (Linné, 1758) & Moineau domestique & House Sparrow & $\mathrm{R}$ & f. \\
\hline Passer griseus (Vieillot, 1817) & Moineau gris & $\begin{array}{l}\text { Grey-headed } \\
\text { Sparrow }\end{array}$ & $\mathrm{R}$ & f. \\
\hline \multicolumn{5}{|l|}{ Ploceidae } \\
\hline Ploceus nigerrimus Vieillot, 1819 & $\begin{array}{l}\text { Tisserin noir de } \\
\text { Vieillot }\end{array}$ & Vieillot's Black & $\mathrm{R}$ & f. \\
\hline Ploceus cucullatus (Muller, 1776) & Tisserin gendarme & Village Weaver & $\mathrm{R}$ & f. \\
\hline Quelea erythrops (Hartlaub, 1848) & $\begin{array}{l}\text { Travailleur à tête } \\
\text { rouge }\end{array}$ & $\begin{array}{l}\text { Red-headed } \\
\text { Quelea }\end{array}$ & M & f. \\
\hline Euplectes afer (J. F. Gmelin, 1789) & Euplecte vorabé & $\begin{array}{l}\text { Yellow-crowned } \\
\text { Bishop }\end{array}$ & $\mathrm{R}$ & f. \\
\hline Euplectes macrourus (J. F. Gmelin, 1789) & Euplecte à dos d'or & $\begin{array}{l}\text { Yellow-mantled } \\
\text { Widowbird }\end{array}$ & $\mathrm{R}$ & f. \\
\hline \multicolumn{5}{|l|}{ Estrildidae } \\
\hline Pyrenestes ostrinus (Vieillot, 1805) & Pyréneste ponceau & $\begin{array}{l}\text { Black-bellied } \\
\text { Seed-cracker }\end{array}$ & $\mathrm{R}$ & $\mathrm{F}$ \\
\hline Spermophaga haematina (Vieillot, 1807) & Sénégali sanguin & Bluebill & $\mathrm{R}$ & $\mathrm{F}$ \\
\hline Estrilda melpoda (Vieillot, 1817) & $\begin{array}{l}\text { Astrild à joues } \\
\text { orange }\end{array}$ & $\begin{array}{l}\text { Orange-cheeked } \\
\text { Waxbill }\end{array}$ & $\mathrm{R}$ & f. \\
\hline Sporaeginthus subflavus (Vieillot, 1819) & Bengali zébré & Zebra Waxbill & $\mathrm{R}$ & f. \\
\hline Ortygospiza atricollis (Vieillot, 1817) & $\begin{array}{l}\text { Astrild-caille à } \\
\text { lunettes }\end{array}$ & Quail Finch & $\mathrm{R}$ & f. \\
\hline Lonchura cucullata (Swainson, 1837) & Capucin nonnette & Bronze Mannikin & $\mathrm{R}$ & f. \\
\hline Lonchura bicolor (Fraser, 1843) & Capucin bicolore & $\begin{array}{l}\text { Red-backed } \\
\text { Mannikin }\end{array}$ & $\mathrm{R}$ & f. \\
\hline Lonchura fringilloides (Lafresnaye, 1835) & Capucin pie & Magpie Mannikin & $\mathrm{R}$ & f. \\
\hline \multicolumn{5}{|l|}{ Viduidae } \\
\hline Vidua macroura (Pallas, 1764) & Veuve dominicaine & $\begin{array}{l}\text { Pin-tailed } \\
\text { Whydah }\end{array}$ & $\mathrm{R}$ & f. \\
\hline
\end{tabular}


S. G. K. ODOUKPE et H. K. YAOKOKORE-BEIBRO / Int. J. Biol. Chem. Sci. 8(4): 1458-1480, 2014

Tableau 2 : Bilan global des observations.

\begin{tabular}{|c|c|c|c|c|c|c|c|c|c|c|c|c|}
\hline \multirow{2}{*}{ Stades } & \multirow{2}{*}{ NE } & \multirow{2}{*}{$\mathbf{A b}$} & \multirow{2}{*}{$\mathbf{H}^{\prime}$} & \multirow{2}{*}{$\mathbf{J}$} & \multicolumn{2}{|c|}{ D } & \multicolumn{2}{|c|}{$\mathbf{R}$} & \multicolumn{2}{|c|}{$\mathbf{R a}$} & \multicolumn{2}{|c|}{ Ac } \\
\hline & & & & & NI & $\% \mathrm{NI}$ & NI & $\% \mathrm{NI}$ & NI & $\% \mathrm{NI}$ & NI & $\% \mathrm{NI}$ \\
\hline TS & 100 & 40556 & 2,2 & 0,48 & 30264 & 74,63 & 5622 & 13,86 & 3184 & 7,85 & 1486 & 3,59 \\
\hline SL & 51 & 1749 & 2,99 & 0,76 & 1051 & 60,09 & 505 & 28,87 & 165 & 9,43 & 28 & 1,6 \\
\hline SS & 65 & 6904 & 2,16 & 0,52 & 4526 & 65,56 & 1820 & 26,36 & 427 & 6,18 & 131 & 1,9 \\
\hline SV & 79 & 2833 & 3,17 & 0,72 & 1639 & 57,85 & 771 & 27,21 & 351 & 12,39 & 72 & 2,54 \\
\hline SRI & 60 & 11778 & 1,56 & 0,38 & 10519 & 89,77 & 437 & 3,73 & 523 & 4,46 & 239 & 2,04 \\
\hline SRM & 61 & 11995 & 1,64 & 0,4 & 10697 & 89,18 & 602 & 5,02 & 414 & 3,45 & 282 & 2,35 \\
\hline SPR & 50 & 5357 & 1,72 & 0,44 & 4551 & 84,95 & 491 & 9,17 & 163 & 3,04 & 152 & 2,84 \\
\hline
\end{tabular}

TS : Tous les stades ; SL : Stade Labour ; SS : Stade Semis ; SV : Stade végétatif ; SRI : Stade Riz Immature ; SRM : Stade Riz Mature ;

SPR : Stade Post-Récolte ; NE : Nombre d'espèces ; Ab : Abondance ; H' : Indice de diversité de Shannon-Weaver ; J : Indice d'Equitabilité :

$\mathrm{NI}$ : Nombre d'Individus ; \%NI : Pourcentage d'abondance ; D : Dominante ; R : régulière ; Ra : Rare ; Ac : Accidentelle

Tableau 3: Bilan des observations sur les rizières de février 2011 à février 2013.

\begin{tabular}{|c|c|c|c|c|c|c|c|c|c|c|c|c|c|c|}
\hline \multirow{2}{*}{ Espèces } & \multicolumn{2}{|c|}{ TS } & \multicolumn{2}{|c|}{$\mathbf{S L}$} & \multicolumn{2}{|c|}{ SS } & \multicolumn{2}{|c|}{ SV } & \multicolumn{2}{|c|}{ SRI } & \multicolumn{2}{|c|}{ SRM } & \multicolumn{2}{|c|}{ SPR } \\
\hline & $\mathbf{A b}$ & $\mathbf{F r}$ & $\mathbf{A b}$ & $\mathbf{F r}$ & $\mathbf{A b}$ & Fr & $\mathbf{A b}$ & Fr & $\mathbf{A b}$ & $\mathbf{F r}$ & $\mathbf{A b}$ & Fr & $\mathbf{A b}$ & Fr \\
\hline Tachybaptus ruficollis & 3 & 0,01 & 0 & 0,00 & 0 & 0,00 & 3 & 0,11 & 0 & 0,00 & 0 & 0,00 & 0 & 0,00 \\
\hline $\begin{array}{l}\text { Phalacrocorax } \\
\text { africanus }\end{array}$ & 5 & 0,01 & 0 & 0,00 & 0 & 0,00 & 4 & 0,14 & 1 & 0,01 & 0 & 0,00 & 0 & 0,00 \\
\hline Ixobrychus minutus & 40 & 0,10 & 0 & 0,00 & 0 & 0,00 & 11 & 0,39 & 17 & 0,15 & 10 & 0,08 & 2 & 0,04 \\
\hline Nycticorax nycticorax & 2 & 0,00 & 0 & 0,00 & 1 & 0,01 & 0 & 0,00 & 0 & 0,00 & 0 & 0,00 & 1 & 0,02 \\
\hline
\end{tabular}


S. G. K. ODOUKPE et H. K. YAOKOKORE-BEIBRO / Int. J. Biol. Chem. Sci. 8(4): 1458-1480, 2014

\begin{tabular}{|c|c|c|c|c|c|c|c|c|c|c|c|c|c|c|}
\hline \multirow{2}{*}{ Espèces } & \multicolumn{2}{|c|}{ TS } & \multicolumn{2}{|c|}{ SL } & \multicolumn{2}{|c|}{ SS } & \multicolumn{2}{|c|}{ SV } & \multicolumn{2}{|c|}{ SRI } & \multicolumn{2}{|c|}{ SRM } & \multicolumn{2}{|c|}{ SPR } \\
\hline & $\mathbf{A b}$ & $\mathbf{F r}$ & $\mathbf{A b}$ & $\mathbf{F r}$ & $\mathbf{A b}$ & Fr & $\mathbf{A b}$ & $\mathbf{F r}$ & $\mathbf{A b}$ & $\mathbf{F r}$ & $\mathbf{A b}$ & Fr & $\mathbf{A b}$ & $\mathbf{F r}$ \\
\hline Ardeola ralloides & 193 & 0,48 & 15 & 0,86 & 75 & 1,09 & 79 & 2,79 & 13 & 0,11 & 8 & 0,07 & 3 & 0,06 \\
\hline Bubulcus ibis & 847 & 2,09 & 281 & 16,07 & 300 & 4,35 & 245 & 8,65 & 2 & 0,02 & 1 & 0,01 & 18 & 0,34 \\
\hline Butorides striata & 16 & 0,04 & 0 & 0,00 & 2 & 0,03 & 6 & 0,21 & 4 & 0,03 & 2 & 0,02 & 2 & 0,04 \\
\hline Egretta ardesiaca & 1 & 0,00 & 0 & 0,00 & 0 & 0,00 & 1 & 0,04 & 0 & 0,00 & 0 & 0,00 & 0 & 0,00 \\
\hline Egretta gularis & 7 & 0,02 & 0 & 0,00 & 0 & 0,00 & 7 & 0,25 & 0 & 0,00 & 0 & 0,00 & 0 & 0,00 \\
\hline Egretta garzetta & 68 & 0,17 & 0 & 0,00 & 5 & 0,07 & 50 & 1,76 & 3 & 0,03 & 10 & 0,08 & 0 & 0,00 \\
\hline Egretta intermedia & 79 & 0,19 & 8 & 0,46 & 26 & 0,38 & 33 & 1,16 & 4 & 0,03 & 2 & 0,02 & 6 & 0,11 \\
\hline Egretta alba & 230 & 0,57 & 60 & 3,43 & 43 & 0,62 & 90 & 3,18 & 5 & 0,04 & 12 & 0,10 & 20 & 0,37 \\
\hline Ardea purpurea & 2 & 0,00 & 0 & 0,00 & 0 & 0,00 & 2 & 0,07 & 0 & 0,00 & 0 & 0,00 & 0 & 0,00 \\
\hline Ardea cinerea & 1 & 0,00 & 0 & 0,00 & 0 & 0,00 & 1 & 0,04 & 0 & 0,00 & 0 & 0,00 & 0 & 0,00 \\
\hline Ardea melanocephala & 2 & 0,00 & 1 & 0,06 & 1 & 0,01 & 0 & 0,00 & 0 & 0,00 & 0 & 0,00 & 0 & 0,00 \\
\hline Ciconia episcopus & 19 & 0,05 & 4 & 0,23 & 10 & 0,14 & 5 & 0,18 & 0 & 0,00 & 0 & 0,00 & 0 & 0,00 \\
\hline Plegadis falcinellus & 2 & 0,00 & 0 & 0,00 & 1 & 0,01 & 1 & 0,04 & 0 & 0,00 & 0 & 0,00 & 0 & 0,00 \\
\hline Dendrocygna viduata & 361 & 0,89 & 3 & 0,17 & 156 & 2,26 & 171 & 6,04 & 18 & 0,15 & 8 & 0,07 & 5 & 0,09 \\
\hline Plectropterus gambensis & 1 & 0,00 & 0 & 0,00 & 1 & 0,01 & 0 & 0,00 & 0 & 0,00 & 0 & 0,00 & 0 & 0,00 \\
\hline Nettapus auritus & 2 & 0,00 & 0 & 0,00 & 0 & 0,00 & 2 & 0,07 & 0 & 0,00 & 0 & 0,00 & 0 & 0,00 \\
\hline Elanus caeruleus & 7 & 0,02 & 0 & 0,00 & 1 & 0,01 & 1 & 0,04 & 2 & 0,02 & 2 & 0,02 & 1 & 0,02 \\
\hline Milvus migrans & 341 & 0,84 & 116 & 6,63 & 194 & 2,81 & 27 & 0,95 & 2 & 0,02 & 1 & 0,01 & 1 & 0,02 \\
\hline Circus pygargus & 2 & 0,00 & 0 & 0,00 & 0 & 0,00 & 0 & 0,00 & 1 & 0,01 & 1 & 0,01 & 0 & 0,00 \\
\hline Circus aeruginosus & 1 & 0,00 & 0 & 0,00 & 0 & 0,00 & 0 & 0,00 & 1 & 0,01 & 0 & 0,00 & 0 & 0,00 \\
\hline Accipiter badius & 2 & 0,00 & 0 & 0,00 & 0 & 0,00 & 1 & 0,04 & 1 & 0,01 & 0 & 0,00 & 0 & 0,00 \\
\hline
\end{tabular}


S. G. K. ODOUKPE et H. K. YAOKOKORE-BEIBRO / Int. J. Biol. Chem. Sci. 8(4): 1458-1480, 2014

\begin{tabular}{|c|c|c|c|c|c|c|c|c|c|c|c|c|c|c|}
\hline \multirow{2}{*}{ Espèces } & \multicolumn{2}{|c|}{ TS } & \multicolumn{2}{|c|}{ SL } & \multicolumn{2}{|c|}{ SS } & \multicolumn{2}{|c|}{ SV } & \multicolumn{2}{|c|}{ SRI } & \multicolumn{2}{|c|}{ SRM } & \multicolumn{2}{|c|}{ SPR } \\
\hline & $\mathbf{A b}$ & $\mathbf{F r}$ & $\mathbf{A b}$ & Fr & $\mathbf{A b}$ & Fr & $\mathbf{A b}$ & $\mathbf{F r}$ & $\mathbf{A b}$ & $\mathbf{F r}$ & $\mathbf{A b}$ & $\mathbf{F r}$ & $\mathbf{A b}$ & $\mathbf{F r}$ \\
\hline Falco ardosiaceus & 7 & 0,02 & 3 & 0,17 & 3 & 0,04 & 1 & 0,04 & 0 & 0,00 & 0 & 0,00 & 0 & 0,00 \\
\hline Crex egregia & 33 & 0,08 & 4 & 0,23 & 2 & 0,03 & 6 & 0,21 & 6 & 0,05 & 10 & 0,08 & 5 & 0,09 \\
\hline Amaurornis flavirostra & 95 & 0,23 & 4 & 0,23 & 18 & 0,26 & 17 & 0,60 & 26 & 0,22 & 22 & 0,18 & 8 & 0,15 \\
\hline Porphyrio alleni & 79 & 0,19 & 0 & 0,00 & 2 & 0,03 & 10 & 0,35 & 40 & 0,34 & 25 & 0,21 & 2 & 0,04 \\
\hline Gallinula angulata & 77 & 0,19 & 1 & 0,06 & 1 & 0,01 & 62 & 2,19 & 11 & 0,09 & 2 & 0,02 & 0 & 0,00 \\
\hline Balearica pavonina & 2 & 0,00 & 0 & 0,00 & 0 & 0,00 & 2 & 0,07 & 0 & 0,00 & 0 & 0,00 & 0 & 0,00 \\
\hline Actophilornis africanus & 869 & 2,14 & 97 & 5,55 & 402 & 5,82 & 250 & 8,82 & 89 & 0,76 & 23 & 0,19 & 8 & 0,15 \\
\hline Rostratula benghalensis & 65 & 0,16 & 10 & 0,57 & 17 & 0,25 & 18 & 0,64 & 4 & 0,03 & 11 & 0,09 & 5 & 0,09 \\
\hline Charadrius hiaticula & 73 & 0,18 & 11 & 0,63 & 28 & 0,41 & 20 & 0,71 & 12 & 0,10 & 2 & 0,02 & 0 & 0,00 \\
\hline Charadrius forbesi & 13 & 0,03 & 0 & 0,00 & 0 & 0,00 & 0 & 0,00 & 0 & 0,00 & 8 & 0,07 & 5 & 0,09 \\
\hline Pluvialis dominica & 4 & 0,01 & 0 & 0,00 & 0 & 0,00 & 4 & 0,14 & 0 & 0,00 & 0 & 0,00 & 0 & 0,00 \\
\hline Vanellus senegalus & 2 & 0,00 & 1 & 0,06 & 1 & 0,01 & 0 & 0,00 & 0 & 0,00 & 0 & 0,00 & 0 & 0,00 \\
\hline Vanellus spinosus & 59 & 0,15 & 12 & 0,69 & 35 & 0,51 & 12 & 0,42 & 0 & 0,00 & 0 & 0,00 & 0 & 0,00 \\
\hline Calidris canutus & 3 & 0,01 & 0 & 0,00 & 0 & 0,00 & 3 & 0,11 & 0 & 0,00 & 0 & 0,00 & 0 & 0,00 \\
\hline Calidris minuta & 3 & 0,01 & 0 & 0,00 & 2 & 0,03 & 1 & 0,04 & 0 & 0,00 & 0 & 0,00 & 0 & 0,00 \\
\hline Calidris fuscicollis & 1 & 0,00 & 0 & 0,00 & 0 & 0,00 & 1 & 0,04 & 0 & 0,00 & 0 & 0,00 & 0 & 0,00 \\
\hline Calidris melanotos & 1 & 0,00 & 0 & 0,00 & 1 & 0,01 & 0 & 0,00 & 0 & 0,00 & 0 & 0,00 & 0 & 0,00 \\
\hline Calidris ferruginea & 18 & 0,04 & 3 & 0,17 & 12 & 0,17 & 3 & 0,11 & 0 & 0,00 & 0 & 0,00 & 0 & 0,00 \\
\hline Gallinago gallinago & 53 & 0,13 & 0 & 0,00 & 1 & 0,01 & 6 & 0,21 & 13 & 0,11 & 22 & 0,18 & 11 & 0,21 \\
\hline Numenius phaeopus & 1 & 0,00 & 0 & 0,00 & 0 & 0,00 & 1 & 0,04 & 0 & 0,00 & 0 & 0,00 & 0 & 0,00 \\
\hline Tringa totanus & 1 & 0,00 & 0 & 0,00 & 0 & 0,00 & 1 & 0,04 & 0 & 0,00 & 0 & 0,00 & 0 & 0,00 \\
\hline & & & & & & & & & & & & & & \\
\hline
\end{tabular}


S. G. K. ODOUKPE et H. K. YAOKOKORE-BEIBRO / Int. J. Biol. Chem. Sci. 8(4): 1458-1480, 2014

\begin{tabular}{|c|c|c|c|c|c|c|c|c|c|c|c|c|c|c|}
\hline \multirow{2}{*}{ Espèces } & \multicolumn{2}{|c|}{ TS } & \multicolumn{2}{|c|}{ SL } & \multicolumn{2}{|c|}{ SS } & \multicolumn{2}{|c|}{ SV } & \multicolumn{2}{|c|}{ SRI } & \multicolumn{2}{|c|}{ SRM } & \multicolumn{2}{|c|}{ SPR } \\
\hline & $\mathbf{A b}$ & $\mathbf{F r}$ & $\mathbf{A b}$ & $\mathbf{F r}$ & $\mathbf{A b}$ & Fr & $\mathbf{A b}$ & $\mathbf{F r}$ & $\mathbf{A b}$ & $\mathbf{F r}$ & $\mathbf{A b}$ & Fr & $\mathbf{A b}$ & $\mathbf{F r}$ \\
\hline Tringa stagnatilis & 2 & 0,00 & 0 & 0,00 & 0 & 0,00 & 1 & 0,04 & 1 & 0,01 & 0 & 0,00 & 0 & 0,00 \\
\hline Tringa nebularia & 13 & 0,03 & 0 & 0,00 & 5 & 0,07 & 4 & 0,14 & 3 & 0,03 & 1 & 0,01 & 0 & 0,00 \\
\hline Tringa ochropus & 1 & 0,00 & 0 & 0,00 & 0 & 0,00 & 0 & 0,00 & 1 & 0,01 & 0 & 0,00 & 0 & 0,00 \\
\hline Tringa glareola & 274 & 0,68 & 42 & 2,40 & 127 & 1,84 & 52 & 1,84 & 31 & 0,26 & 16 & 0,13 & 6 & 0,11 \\
\hline Actitis hypoleucos & 43 & 0,11 & 2 & 0,11 & 17 & 0,25 & 14 & 0,49 & 6 & 0,05 & 2 & 0,02 & 2 & 0,04 \\
\hline Turtur tympanistria & 3 & 0,01 & 0 & 0,00 & 2 & 0,03 & 0 & 0,00 & 0 & 0,00 & 0 & 0,00 & 1 & 0,02 \\
\hline Turtur afer & 78 & 0,19 & 21 & 1,20 & 23 & 0,33 & 10 & 0,35 & 7 & 0,06 & 6 & 0,05 & 11 & 0,21 \\
\hline $\begin{array}{l}\text { Streptopelia } \\
\text { semitorquata }\end{array}$ & 1999 & 4,93 & 205 & 11,72 & 638 & 9,24 & 23 & 0,81 & 0 & 0,00 & 3 & 0,03 & 1130 & 21,09 \\
\hline $\begin{array}{l}\text { Streptopelia } \\
\text { senegalensis }\end{array}$ & 53 & 0,13 & 5 & 0,29 & 28 & 0,41 & 9 & 0,32 & 0 & 0,00 & 1 & 0,01 & 10 & 0,19 \\
\hline Chrysococcyx caprius & 10 & 0,02 & 0 & 0,00 & 0 & 0,00 & 5 & 0,18 & 2 & 0,02 & 3 & 0,03 & 0 & 0,00 \\
\hline Centropus grillii & 5 & 0,01 & 0 & 0,00 & 0 & 0,00 & 2 & 0,07 & 2 & 0,02 & 0 & 0,00 & 1 & 0,02 \\
\hline Centropus senegalensis & 3 & 0,01 & 0 & 0,00 & 0 & 0,00 & 2 & 0,07 & 1 & 0,01 & 0 & 0,00 & 0 & 0,00 \\
\hline Cypsiurus parvus & 89 & 0,22 & 0 & 0,00 & 7 & 0,10 & 12 & 0,42 & 31 & 0,26 & 39 & 0,33 & 0 & 0,00 \\
\hline Apus affinis & 2 & 0,00 & 0 & 0,00 & 0 & 0,00 & 2 & 0,07 & 0 & 0,00 & 0 & 0,00 & 0 & 0,00 \\
\hline Halcyon malimbica & 2 & 0,00 & 1 & 0,06 & 1 & 0,01 & 0 & 0,00 & 0 & 0,00 & 0 & 0,00 & 0 & 0,00 \\
\hline Halcyon senegalensis & 51 & 0,13 & 5 & 0,29 & 13 & 0,19 & 11 & 0,39 & 7 & 0,06 & 5 & 0,04 & 10 & 0,19 \\
\hline Ceyx pictus & 4 & 0,01 & 1 & 0,06 & 3 & 0,04 & 0 & 0,00 & 0 & 0,00 & 0 & 0,00 & 0 & 0,00 \\
\hline Alcedo cristata & 22 & 0,05 & 1 & 0,06 & 3 & 0,04 & 9 & 0,32 & 2 & 0,02 & 2 & 0,02 & 5 & 0,09 \\
\hline Ceryle rudis & 12 & 0,03 & 0 & 0,00 & 0 & 0,00 & 11 & 0,39 & 0 & 0,00 & 1 & 0,01 & 0 & 0,00 \\
\hline
\end{tabular}


S. G. K. ODOUKPE et H. K. YAOKOKORE-BEIBRO / Int. J. Biol. Chem. Sci. 8(4): 1458-1480, 2014

\begin{tabular}{|c|c|c|c|c|c|c|c|c|c|c|c|c|c|c|}
\hline \multirow{2}{*}{ Espèces } & \multicolumn{2}{|c|}{ TS } & \multicolumn{2}{|c|}{ SL } & \multicolumn{2}{|c|}{ SS } & \multicolumn{2}{|c|}{ SV } & \multicolumn{2}{|c|}{ SRI } & \multicolumn{2}{|c|}{ SRM } & \multicolumn{2}{|c|}{ SPR } \\
\hline & $\mathbf{A b}$ & $\mathbf{F r}$ & $\mathbf{A b}$ & Fr & $\mathbf{A b}$ & Fr & $\mathbf{A b}$ & $\mathbf{F r}$ & $\mathbf{A b}$ & $\mathbf{F r}$ & $\mathbf{A b}$ & Fr & $\mathbf{A b}$ & Fr \\
\hline Merops pusillus & 27 & 0,07 & 9 & 0,51 & 7 & 0,10 & 5 & 0,18 & 4 & 0,03 & 1 & 0,01 & 1 & 0,02 \\
\hline Riparia riparia & 1 & 0,00 & 0 & 0,00 & 0 & 0,00 & 0 & 0,00 & 0 & 0,00 & 1 & 0,01 & 0 & 0,00 \\
\hline Hirundo semirufa & 19 & 0,05 & 3 & 0,17 & 2 & 0,03 & 10 & 0,35 & 3 & 0,03 & 1 & 0,01 & 0 & 0,00 \\
\hline Hirundo abyssinica & 29 & 0,07 & 8 & 0,46 & 13 & 0,19 & 8 & 0,28 & 0 & 0,00 & 0 & 0,00 & 0 & 0,00 \\
\hline Hirundo aethiopica & 29 & 0,07 & 6 & 0,34 & 8 & 0,12 & 13 & 0,46 & 0 & 0,00 & 2 & 0,02 & 0 & 0,00 \\
\hline Hirundo rustica & 99 & 0,24 & 0 & 0,00 & 0 & 0,00 & 0 & 0,00 & 46 & 0,39 & 47 & 0,39 & 6 & 0,11 \\
\hline Motacilla flava & 215 & 0,53 & 41 & 2,34 & 112 & 1,62 & 19 & 0,67 & 14 & 0,12 & 19 & 0,16 & 10 & 0,19 \\
\hline Anthus leucophrys & 122 & 0,30 & 46 & 2,63 & 39 & 0,56 & 20 & 0,71 & 4 & 0,03 & 1 & 0,01 & 12 & 0,22 \\
\hline Macronyx croceus & 60 & 0,15 & 12 & 0,69 & 14 & 0,20 & 12 & 0,42 & 5 & 0,04 & 6 & 0,05 & 11 & 0,21 \\
\hline Pycnonotus barbatus & 11 & 0,03 & 3 & 0,17 & 1 & 0,01 & 0 & 0,00 & 2 & 0,02 & 3 & 0,03 & 2 & 0,04 \\
\hline Saxicola rubetra & 5 & 0,01 & 2 & 0,11 & 3 & 0,04 & 0 & 0,00 & 0 & 0,00 & 0 & 0,00 & 0 & 0,00 \\
\hline $\begin{array}{l}\text { Acrocephalus } \\
\text { schoenobaenus }\end{array}$ & 3 & 0,01 & 0 & 0,00 & 0 & 0,00 & 0 & 0,00 & 1 & 0,01 & 2 & 0,02 & 0 & 0,00 \\
\hline Cisticola erythrops & 19 & 0,05 & 0 & 0,00 & 6 & 0,09 & 5 & 0,18 & 0 & 0,00 & 6 & 0,05 & 2 & 0,04 \\
\hline Cisticola galactotes & 244 & 0,60 & 20 & 1,14 & 24 & 0,35 & 46 & 1,62 & 56 & 0,48 & 69 & 0,58 & 29 & 0,54 \\
\hline Cisticola brachypterus & 37 & 0,09 & 3 & 0,17 & 0 & 0,00 & 6 & 0,21 & 5 & 0,04 & 14 & 0,12 & 9 & 0,17 \\
\hline Prinia subflava & 39 & 0,10 & 7 & 0,40 & 5 & 0,07 & 8 & 0,28 & 4 & 0,03 & 8 & 0,07 & 7 & 0,13 \\
\hline Cinnyris cupreus & 9 & 0,02 & 0 & 0,00 & 0 & 0,00 & 1 & 0,04 & 4 & 0,03 & 4 & 0,03 & 0 & 0,00 \\
\hline Lanius collaris & 12 & 0,03 & 6 & 0,34 & 4 & 0,06 & 2 & 0,07 & 0 & 0,00 & 0 & 0,00 & 0 & 0,00 \\
\hline Corvus albus & 42 & 0,10 & 21 & 1,20 & 21 & 0,30 & 0 & 0,00 & 0 & 0,00 & 0 & 0,00 & 0 & 0,00 \\
\hline Passer domesticus & 1 & 0,00 & 0 & 0,00 & 1 & 0,01 & 0 & 0,00 & 0 & 0,00 & 0 & 0,00 & 0 & 0,00 \\
\hline
\end{tabular}


S. G. K. ODOUKPE et H. K. YAOKOKORE-BEIBRO / Int. J. Biol. Chem. Sci. 8(4): 1458-1480, 2014

\begin{tabular}{|c|c|c|c|c|c|c|c|c|c|c|c|c|c|c|}
\hline \multirow{2}{*}{ Espèces } & \multicolumn{2}{|c|}{ TS } & \multicolumn{2}{|c|}{ SL } & \multicolumn{2}{|c|}{ SS } & \multicolumn{2}{|c|}{ SV } & \multicolumn{2}{|c|}{ SRI } & \multicolumn{2}{|c|}{ SRM } & \multicolumn{2}{|c|}{ SPR } \\
\hline & $\mathbf{A b}$ & $\mathbf{F r}$ & $\mathbf{A b}$ & $\mathbf{F r}$ & $\mathbf{A b}$ & $\mathbf{F r}$ & $\mathbf{A b}$ & $\mathbf{F r}$ & $\mathbf{A b}$ & $\mathbf{F r}$ & $\mathbf{A b}$ & $\mathbf{F r}$ & $\mathbf{A b}$ & $\mathbf{F r}$ \\
\hline Passer griseus & 48 & 0,12 & 11 & 0,63 & 17 & 0,25 & 10 & 0,35 & 4 & 0,03 & 5 & 0,04 & 1 & 0,02 \\
\hline Ploceus nigerrimus & 87 & 0,21 & 0 & 0,00 & 0 & 0,00 & 0 & 0,00 & 22 & 0,19 & 55 & 0,46 & 10 & 0,19 \\
\hline Ploceus cucullatus & 8837 & 21,79 & 49 & 2,80 & 282 & 4,08 & 100 & 3,53 & 2734 & 23,33 & 2952 & 24,61 & 2720 & 50,77 \\
\hline Quelea erythrops & 16209 & 39,97 & 11 & 0,63 & 3486 & 50,49 & 481 & 16,98 & 6273 & 53,53 & 5806 & 48,40 & 152 & 2,84 \\
\hline Euplectes afer & 2201 & 5,43 & 208 & 11,89 & 141 & 2,04 & 252 & 8,90 & 652 & 5,56 & 678 & 5,65 & 270 & 5,04 \\
\hline Euplectes macr & 345 & 0,85 & 56 & 3,20 & 45 & 0,65 & 38 & 1,34 & 110 & 0,94 & 72 & 0,60 & 24 & 0,45 \\
\hline Pyrenestes ostrinus & 20 & 0,05 & 0 & 0,00 & 0 & 0,00 & 2 & 0,07 & 9 & 0,08 & 7 & 0,06 & 2 & 0,04 \\
\hline $\begin{array}{l}\text { Spermophaga } \\
\text { haematina }\end{array}$ & 9 & 0,02 & 0 & 0,00 & 0 & 0,00 & 0 & 0,00 & 0 & 0,00 & 3 & 0,03 & 6 & 0,11 \\
\hline Estrilda melpoda & 1055 & 2,60 & 34 & 1,94 & 17 & 0,25 & 127 & 4,48 & 280 & 2,39 & 383 & 3,19 & 214 & 3,99 \\
\hline Sporaeginthus subflavus & 5 & 0,01 & 0 & 0,00 & 0 & 0,00 & 1 & 0,04 & 2 & 0,02 & 2 & 0,02 & 0 & 0,00 \\
\hline Ortygospiza atricollis & 90 & 0,22 & 17 & 0,97 & 15 & 0,22 & 2 & 0,07 & 4 & 0,03 & 25 & 0,21 & 27 & 0,50 \\
\hline Lonchura cucullata & 3017 & 7,44 & 144 & 8,23 & 81 & 1,17 & 240 & 8,47 & 860 & 7,34 & 1261 & 10,51 & 431 & 8,05 \\
\hline Lonchura bicolor & 120 & 0,30 & 0 & 0,00 & 0 & 0,00 & 6 & 0,21 & 68 & 0,58 & 39 & 0,33 & 7 & 0,13 \\
\hline Lonchura fringilloides & 852 & 2,10 & 81 & 4,63 & 275 & 3,98 & 62 & 2,19 & 157 & 1,34 & 219 & 1,83 & 58 & 1,08 \\
\hline Vidua macroura & 279 & 0,69 & 34 & 1,94 & 77 & 1,12 & 32 & 1,13 & 26 & 0,22 & 43 & 0,36 & 67 & 1,25 \\
\hline & 40556 & & 1749 & & 6904 & & 2833 & & 11718 & & 11995 & & 5357 & \\
\hline
\end{tabular}

TS : Tous les stades ; SL : Stade Labour ; SS : Stade Semis ; SV : Stade végétatif ; SRI : Stade Riz Immature ; SRM : Stade Riz Mature ; SPR : Stade Post-Récolte ; $\mathrm{Ab}$ : Abondance; $\mathrm{Fr}$ : Fréquence relative. 


\section{DISCUSSION}

Les champs de riz de la zone humide de Grand-Bassam abritent une avifaune riche et diversifiée à l'image de ce site Ramsar (Yaokokoré-Béibro et al., 2010). Comparée à l'avifaune de toute la zone de riziculture, il ressort que près de la moitié des oiseaux utilisent directement les rizières pour se nourrir (Odoukpé et al., 2014). Quelques-uns en ont fait leur habitat temporaire et en profitent pour se reproduire assurant ainsi la survie de leur population. En réalité, les champs de riz seraient, de toutes les cultures, l'un des habitats les plus diversifiés en espèces d'oiseaux (Sundar et Subramanya, 2010). Tout comme Elphick (2010), il est constaté que les familles les plus diversifiées, dans les champs de riz, sont celles qui regroupent les hérons, les aigrettes et les oiseaux de rivage. En fait, à certaines périodes, les rizières offrent aux oiseaux d'eau des ressources alimentaires et des habitats semblables aux zones humides naturelles qu'ils affectionnent. C'est d'ailleurs l'une des raisons qui motivent les écologues à considérer les rizières comme des habitats substitutifs des zones humides naturelles en perte, pour les oiseaux d'eau (Fasola et Ruiz, 1996). Certes, les oiseaux d'eau se regroupent dans des familles plus importantes, mais les oiseaux des milieux ouverts comptent le plus grand nombre d'espèces. Les oiseaux les moins représentés sont ceux qui affectionnent les forêts secondaires. De ce fait, Sundar et Subramanya (2010) font remarquer que les espèces d'oiseaux exploitant les champs de riz sont bien représentées par les oiseaux des milieux ouverts et moins représentés par ceux des habitats boisés.

La distribution des espèces d'oiseaux était non aléatoire et s'est effectuée en fonction des différents stades phénologiques de la croissance du riz. Les changements des paramètres physiques et biologiques des rizières expliqueraient cette distribution des oiseaux et des observations similaires sont faites par d'autres auteurs (Nachuha, 2009;
Sundar et Subramanya, 2010). Les champs de riz sont beaucoup plus diversifiés en début de culture du riz qu'en fin de culture. C'est d'ailleurs au stade végétatif que le nombre d'espèces et l'indice de diversité les plus élevés ont été enregistrés. Cette forte diversité d'espèces d'oiseaux en début de culture serait probablement liée à l'habitat clairsemé et faiblement couvert d'eau que présentent les champs de riz. En plus, l'absence de surveillance à cette période favoriserait la visite de toutes les catégories d'espèces pour exploiter la diversité des ressources disponibles. Les plus faibles indices de diversité sont par contre enregistrés à la phase de remplissage et de maturation du riz. Ce constat pourrait être lié à la végétation dense des rizières et à la présence de gardiens à cette période qui limiteraient l'arrivée de certaines catégories d'espèces telles que les rapaces et les oiseaux d'eau dans les champs. Sur la base du peuplement d'oiseaux observé à chaque stade du cycle du riz et des relations révélées par les différentes analyses statistiques, il serait dorénavant convenable de scinder le cycle du riz en quatre phases pour les observations ornithologiques: la phase des labours, la phase de semence et de croissance, la phase de remplissage et de maturation et la phase des récoltes. C'est d'ailleurs la méthode utilisée par Nachuha (2009) dans une étude sur les oiseaux d'eau des champs de riz en Ouganda.

Les rizières de la zone humide de Grand-Bassam attirent de grandes populations d'espèces qui varient d'un stade à un autre. En effet, les cultures de riz permettent aux grands groupes d'oiseaux de s'alimenter en masse (Tréca, 1989). Les plus grands effectifs du peuplement avifaunistique des rizières se sont enregistrés à la phase de remplissage et de maturation du riz. Toutefois, les effectifs du peuplement n'étaient négligeables aux phases de semence et de récolte. Cette forte utilisation des rizières par l'avifaune serait majoritairement liée à la présence de grains de riz à ces différentes phases. En réalité, le 
groupe trophique des granivores constitue l'essentiel du peuplement d'oiseaux des rizières à ces phases. De plus, ce peuplement des granivores est assez bien représenté aux stades des labours et de croissance du riz. Sur l'ensemble du cycle du riz, les rizières semblent donc fournir à ces oiseaux granivores une gamme importante de ressources biologiques. Contrairement aux granivores, les autres groupes trophiques sont relativement moins abondants. Suivant l'importance trophique des différents groupes d'espèces, il est vraisemblable que les oiseaux soient des auxiliaires de l'homme en début de culture et des compétiteurs en fin de cycle. En fait, ils agissent en régulateurs aux pullulations d'invertébrés et de petits vertébrés tout en profitant de cet apport alimentaire appréciable en début de culture. Par contre, à la phase de remplissage et de maturation et dans certaines mesures à la récolte, les granivores qui apprécient le riz deviennent numériquement très importants malgré les gardes qui les pourchassent à l'aide de diverses méthodes (Baouab, 2008).

Les populations d'oiseaux les plus abondantes enregistrées sur l'ensemble du cycle du riz sont inévitablement celles des oiseaux granivores. Les espèces en question sont le Tisserin gendarme Ploceus cucullatus, le Travailleur à tête rouge Quelea erythrops, l'Euplecte vorabé Euplectes afer et le Capucin nonnette Lonchura cucullata. Ce sont toutes des espèces qui ont un régime essentiellement granivore et qui pourraient causer des dégâts aux cultures rizicoles. Toutefois, les populations du Héron garde-bœuf Bubulcus ibis, du Dendrocygne veuf Dendrocygna viduata, du Milan noir Milvus migrans, de la Tourterelle à collier Streptopelia semitorquata et du Jacana à poitrine dorée Actophilornis africana sont abondantes en début de culture $\mathrm{du}$ riz. Suivant le niveau trophique de ces dernières, seuls le Dendrocygne veuf et la Tourterelle à collier pourraient avoir un impact négatif sur la production du riz.

\section{Conclusion}

Les travaux effectués dans la zone de riziculture de Grand-Bassam exposent les premières données sur les oiseaux rencontrés dans les champs de riz en Côte d'Ivoire. Cette liste liminaire donne un aperçu de la richesse de l'avifaune des surfaces rizicoles. La structure du peuplement est majoritairement dominée par les oiseaux des milieux ouverts et les oiseaux d'eau. Pour ces derniers, les rizières pourraient jouer un rôle primordial dans la gestion durable de leur population. Suivant leurs divers besoins biologiques, les espèces d'oiseaux se distribuent en fonction des différentes phases de la culture de riz. Ainsi, les champs de riz regroupent toutes les catégories trophiques d'oiseaux en début de production du riz. Par contre, en fin de production, les champs de riz deviennent exclusivement l'apanage des seuls oiseaux granivores. Pour mieux connaître les oiseaux qui exploitent les champs de riz en vue de résoudre les problèmes de déprédation et/ou assurer la gestion durable des populations d'oiseaux d'eau, d'autres études similaires devraient s'effectuer dans les zones favorables à la riziculture en Côte d'Ivoire.

\section{REMERCIEMENTS}

Nous sommes reconnaissants à $\mathrm{M}$. Kouamé et à l'ensemble des paysans de la zone de riziculture de Grand-Bassam pour leur coopération et leur disponibilité à nous venir en aide chaque fois qu'il était nécessaire pendant la période de récolte des données.

\section{REFERENCES}

Ahmadi N, Bouman B. 2013. Riz et rizicultures, enjeux économiques, écologiques et scientifiques. Cah. Agric., 22 : 333-335.

Baouab RE. 2008. Composition avifaunistique et fonctionnement des rizières de la province de Sidi Kacem (Maroc). Bulletin de l'Institut Scientifique, Section Sciences de la Vie, 30 : 37-44. 
Bennun L, Dranzoa C, Pomeroy D. 1996. The forest birds of Kenyan and Uganda. Journal of East African Natural History, 85: 23-48.

Bibby C, Martin J, Marsden S. 1998. Birds Survey in Expedition Field Techniques. Royal Geographical Society : London.

Bisby F, Roskov Y, Orrell T, Nicolson D, Paglinawan L, Bailly N, Kirk P, Bourgoin T, Hertum JV. 2008. Species 2000 and ITIS Catalogue of Life : 2008 Annual Checklist. CD-ROM; Species 2000: Reading, U.K.

Borrow N, Demey R. 2001. Birds of Western Africa. HELM Identification guides: London.

Borrow N, Demey R. 2008. Guide des Oiseaux de l'Afrique de l'Ouest. Delachaux et Niestlé : Paris.

Brou Y. 1997. Analyse et dynamique de la pluviométrie en milieu forestier ivoirien. Thèse de Doctorat $3^{\text {ème }}$ cycle, Université de Cocody, Abidjan, p. 200.

Chappuis C. 2000. African Bird Sounds. Birds of North, West and Central Africa. Livrette et 15 CD. Société d'Études Ornithologiques de France : Paris.

Cho DO. 2007. The evolution and resolution of conflicts on Saemangeum Reclamation Project. Ocean and Coastal Management, 50: $930-944$.

Eadie JM, Elphick CS, Reinecke KJ, Miller MR. 2008. Wildlife values of North American ricelands. In Conservation in Ricelands of North America, Manley SW (ed). The Rice Foundation: Arkansas; 790.

Elphick CS. 2000. Functional equivalency between rice fields and seminatural wetland habitats. Conservation Biology, 14: 181-191.

Elphick CS. 2010. Why study birds in rice fields? Waterbirds, 33(1): 1-7.

Elphick CS, Baicich P, Parsons KC, Fasola M, Mugica L. 2010. The future for research on waterbirds in rice fields. Waterbirds, 33(1): 231-243.
Fasola M, Ruiz X. 1996. The value of rice fields as substitutes for natural wetlands for waterbirds in the Mediterranean region. Colonial Waterbirds, 19(1): 122128.

Guillaumet JL, Adjanohoun E. 1971. La végétation de la Côte d'Ivoire. In $L e$ Milieu Naturel de la Côte d'Ivoire, Avenard JM, Eldin M, Girard G, Sircoulon J, Touchebeuf P, Guillaumet JL, Adjanohoun E, Perraud A (eds). ORSTOM : Paris ; 157-263.

Maclean JL, Dawe DC, Hardy B, Hettel GP. 2002. Rice Almanac: Source Book for the Most Important Economic Activity of Earth. CABI Publishing: UK, Wallingford.

Nachuha S. 2009. Is waterbirds distribution within rice paddies of eastern Uganda affected by the different stages of rice growing? In Proceedings of the 12th PanAfrican Ornithological Congress 2008, Harebottle DM, Craig AJFK, Anderson MD, Rakotomanana H, Muchai M (eds). Animal Demography Unit: Cape Town; 44-49.

Odoukpé KSG, Yaokokoré Béibro KH, Kouadio KP, Konan EM. 2014. Dynamique du peuplement des Oiseaux d'une riziculture et ses environs dans la zone humide d'importance internationale de Grand-Bassam. J. Appl. Biosci., 79: 6909-6925.

Ormerod SJ, Marshall EJP, Kerby G, Rushton SP. 2003. Meeting the ecological challenges of agricultural change: editor's introduction. Journal of Applied Ecology, 40: 939-946.

Ramade F. 1994. Éléments d'Ecologie : Ecologie Fondamentale $\left(2^{\mathrm{è}}\right.$ edn). Ediscience International: Paris.

Sundar KSG, Subramanya S. 2010. Birds use of rice fields in the Indian Subcontinent. Waterbirds, 33(1) : 44-70.

Thiollay JM. 1986. Structure comparée du peuplement avien des trois sites de forêt 
primaire en Guyane. La Terre et la Vie, 41: 59-105.

Toral GM, Aragonès D, Bustamante J, Figuerola J. 2011. Using landstat images to map habitat availability for waterbirds in rice fields. Ibis, 153: 684-694.

Tréca B. 1989. Les risques de dégâts d'Oiseaux sur les rizières sahéliennes. In Le risque en agriculture, Eldin M, Milleville P (eds). ORSTOM: Paris; 167175.

Wymenga E, Zwarts L. 2010. Use of rice fields by birds in West Africa. Waterbirds, 33(1): 97-104.

Yaokokoré-Béibro KH. 2001. Avifaune des forêts classées de l'Est de la Côte
d'Ivoire: données sur l'écologie des espèces et effet de la déforestation sur les peuplements. Cas des forêts classées de la Béki et de la Bossématié (Abengourou). Thèse de Doctorat, Université de Cocody, Abidjan, p. 245.

Yaokokoré-Béibro KH, N'guessan AM, Odoukpé KSG, Zouzou EJ, N'douba V, Kouassi KP. 2010. Premières données sur les Oiseaux de la zone humide d'importance internationale de GrandBassam (Côte d'Ivoire). Int. J. Biol. Chem. Sci, 4(6): 2169-2180. 